\title{
Aproveitamento dos resíduos da produção de conserva de palmito como substrato para plantas
}

\author{
Maria Helena Fermino ${ }^{1}$; Reinaldo S Gonçalves ${ }^{2}$; Alice Battistinn'; José Ricardo P Silveira ${ }^{1}$; Ângela Cris- \\ tina Busnello ${ }^{1}$; Miriam Trevisam ${ }^{1}$ \\ ${ }^{1}$ FEPAGRO, R. Gonçalves Dias, 570, Menino Deus, 90130-060 Porto Alegre-RS; ${ }^{2}$ UFRGS-Instituto de Química, C. Postal 15049; \\ 91501-970 Porto Alegre-RS; maria-fermino@fepagro.rs.gov.br; reinaldo@iq.ufrgs.br; alice-battistin@fepagro.rs.gov.br; jose-silveira@ \\ fepagro.rs.gov.br; acbusnello@yahoo.com.br; miriamagronomia@gmail.com
}

\section{RESUMO}

Avaliou-se as características físicas e químicas dos resíduos da extração do palmito da palmeira real australiana [Archontophoenix alexandrae (F. Muell.) H. Wendl. \& Drude] com vistas à sua utilização como substrato para plantas. Estudou-se amostras originárias de dois locais, do interior e do litoral, e subdividiu-se as mesmas segundo as partes da planta em folhas, estipe, e "cartucho" (bainhas), sendo analisadas na forma in natura e após sofrer hidrólise ácida. Todas as amostras foram caracterizadas determinando-se a densidade úmida e seca, a porosidade total, o espaço de aeração, a disponibilidade de água, o valor de $\mathrm{pH}$, a condutividade elétrica e o teor total de sais solúveis. Não houve diferença estatística entre as amostras para as características físicas estudadas. As amostras oriundas do litoral não diferiram daquelas obtidas no interior, assim como, as amostras que sofreram hidrólise ácida não diferiram daquelas in natura. Da mesma forma, as partes da planta (folhas, estipe e cartucho) não apresentaram diferenças significativas entre si. No entanto, os resultados das características químicas consideradas não são apropriados para a utilização dos resíduos da indústria de conserva de palmito como substrato para plantas e indicam a necessidade de novos estudos com resíduos provenientes de plantio controlado da palmeira real australiana.

Palavras-chave: Archontophoenix alexandrae, palmeira real australiana, hidrólise ácida, características físicas, características químicas.

\begin{abstract}
Reutilization of wastes from the production of palm heart canning as substrates for plants

A study was held to evaluate the physical and chemical characteristics of wastes from the extraction of the heart of the Australian real palm (Archontophoenix alexandrae), in order to use it as substrates for plants. Samples from inland and from seaside were subdivided into leaves, stipe and "cartridge" (sheaths) and analyzed in the in natura form and after acid hydrolysis. All samples were characterized by humid and dry density, total porosity, aeration space and availability of water, $\mathrm{pH}$, electric conductivity and total content of soluble salts. There was no statistical difference among the samples for the studied physical characteristics. The samples from seaside did not differ from those from inland and the samples that have suffered acid hydrolysis did not differ from those in natura. Also, the parts of the plant (leaves, stipe and cartridge) showed no significant differences among themselves. The results of chemical characteristics showed the utilization of wastes from the production of palm heart canning as substrates for plants is not recommended. Additionally, the results evidenced the need for new studies with waste from controlled planting of Australian real palm.
\end{abstract}

Keywords: Archontophoenix alexandrae, Australian real palm, acid hydrolysis, physical characteristics, chemical characteristics.

(Recebido para publicação em 31 de março de 2009; aceito em 24 de junho de 2010) (Received on March 31, 2009; accepted on June 24, 2010)

\begin{abstract}
$\mathrm{A}^{\mathrm{s}}$ s plantas olerícolas, florestais, frutíferas, ornamentais, medicinais, aromáticas e mesmo culturas industriais como fumo necessitam, em pelo menos algumas fases do seu desenvolvimento, do cultivo em recipientes (bandejas, sacos, vasos, tubetes, entre outros). Ao meio onde se desenvolvem as raízes em substituição ao solo in situ e que portanto, serve de suporte à planta, denominase substrato (Kämpf, 2000).

Existem referências na literatura (Maas \& Adamson, 1975; Worral, 1978; Günther, 1984; Verdonck, 1984; Waller \& Wilson, 1984; Lamanna et al., 1991; Raja Harun et al., 1991; Schmilewski,
\end{abstract}

1991; Miner, 1994; Fermino, 1996; Müller, 2000) do uso de diversos materiais como substrato para plantas, como por exemplo estercos, fibras naturais, lixo urbano, solo, poliuretanos, aguapé, bagaço de cana, "confete" de papel (formulário contínuo), maravalha, cascas de árvores, casca de arroz e até mesmo pneu picado.

Um dos exemplos mais recentes e de grande abrangência no país é a fibra da casca do coco. Até então se constituía num "poluente" do ambiente sendo utilizada em baixa escala pela indústria para confecção de bancos de automóveis. A partir da determinação do potencial do material como substrato, as grandes empresas, que até então cultivavam o coqueiro para obtenção do coco e da água, passaram a implantar áreas de cultivo com o objetivo de obter matéria-prima para as fábricas de substrato. Este substrato tem despertado interesse internacional como substituto de materiais considerados excelentes como a turfa e a fibra de xaxim.

Por outro lado, a Mata Atlântica abriga nove gêneros e trinta e nove espécies de palmeiras, sendo a palmeira juçara (Euterpe edulis Mart.), de onde se obtém o palmito, uma espécie nativa que cresce no substrato inferior da flo- 
resta. A exploração do palmito sempre se deu de forma extrativista, buscando a matéria-prima nas reservas naturais existentes, sem haver preocupação com o repovoamento das áreas exploradas.

Como não poderia deixar de acontecer, a palmeira nativa Euterpe edulis, tornou-se escassa e com grande possibilidade de ser extinta. Com a redução da população desta palmeira, outras espécies passaram a ser estudadas, buscando-se plantas mais precoces e que produzam um produto com a qualidade do palmito obtido com a palmeira nativa. Dentre elas, tem merecido destaque a palmeira real australiana (Archontophoenix alexandrae).

A colheita do palmito é feita após um período médio de desenvolvimento que varia de quatro anos, para a palmeira real australiana, e de até oito anos, para as nativas como a juçara. A cada planta de palmito colhida, extrai-se cerca de $400 \mathrm{~g}$ de palmito comercial, gerando-se aproximadamente $13 \mathrm{~kg}$ de resíduos que incluem estipe, folhas e bainhas. Parte deste material permanece no local da colheita ou do processamento, sem nenhuma finalidade prática, gerando cerca de 20 toneladas por semana de "lixo", conforme dados da empresa Natusol.

Considerando os fatos expostos, o presente estudo teve por objetivo avaliar as características físicas e químicas dos resíduos provenientes da extração do palmito da palmeira real australiana com vistas a sua utilização como substrato para plantas.

\section{MATERIAL E MÉTODOS}

1. Preparo das amostras - O presente estudo foi realizado em laboratório da FEPAGRO, de agosto a dezembro de 2004. Os resíduos oriundos da palmeira real australiana (Archontophoenix alexandrae), foram coletados em dois locais do Rio Grande do Sul: do interior do estado, no município de Vale do Sol onde se localiza a empresa Natusol, e de várias propriedades no município de Torres, no litoral do estado. Desta forma, os resíduos foram divididos em dois grupos segundo a origem: plantas do interior e do litoral, respectivamente.

Os resíduos, após a retirada do palmito, foram ainda segregados em três grupos segundo as partes da planta: folhas, estipe e "cartucho" (bainhas).

Estes resíduos ainda foram utilizados na forma in natura (triturados e secos a $65^{\circ} \mathrm{C}$ ) e após sofrer hidrólise ácida, a $1 \%(\mathrm{v} / \mathrm{v})$ de ácido sulfúrico por 30 minutos de autoclavagem em condições de temperatura de $120^{\circ} \mathrm{C}$ e 1,5 atm de pressão (trituração, hidrólise e secagem a $65^{\circ} \mathrm{C}$ ).

2. Métodos para a caracterização física e química - Para análise das características físicas determinou-se a densidade úmida e seca, a porosidade total, o espaço de aeração e a disponibilidade de água. As características químicas analisadas foram valor de $\mathrm{pH}$, condutividade elétrica e teor total de sais solúveis.

Para a obtenção da densidade de volume foi utilizado o método adotado pela União das Entidades Alemãs de Pesquisas Agrícolas (VDLUFA), para análise de substratos hortícolas (Hoffmann, 1970; Röber \& Schaller, 1985).

Preencher uma proveta plástica transparente e graduada, de $500 \mathrm{~mL}$, com o substrato. Após, esta proveta é deixada cair, sob a ação do seu próprio peso, de uma altura de $10 \mathrm{~cm}$ por dez vezes consecutivas. Com auxílio de uma espátula nivela-se a superfície levemente, e lê-se o volume obtido (em mL). Em seguida pesa-se o material úmido (em g) e leva-se à estufa para secagem a $65^{\circ} \mathrm{C}$ até peso constante (ou 48 horas).

Os valores das densidades de volume foram obtidos aplicando-se as seguintes fórmulas:

$$
\begin{aligned}
& \text { D. úmida }\left(\mathrm{kg} \mathrm{m}^{-3}\right)=\frac{\text { Peso úmido }(\mathrm{g})}{\text { Volume }(\mathrm{mL})} * 1000 \\
& \text { Matéria } \sec a(\%)=1-\frac{(\text { Peso úmido }- \text { Peso } \sec o)}{\text { Peso úmido }} * 100 \\
& \text { D. } \sec a\left(\mathrm{~kg} \mathrm{~m}^{-3}\right)=\frac{\text { D. úmida }\left(\mathrm{kg} \mathrm{m}^{-3}\right) * \text { Matéria } \sec a(\%)}{100}
\end{aligned}
$$

A determinação da porosidade total, espaço de aeração, água facilmente disponível, água tamponante e disponível foi realizada através de curvas de retenção de água nas tensões de 0 , 10,50 , e $100 \mathrm{~cm}$ de altura de coluna de água, correspondendo às pressões de $0,10,50$ e $100 \mathrm{hPa}$ (De Boodt \& Verdonck, 1972). Funis de Bütner com placas porosas ( $1 / 2$ bar) foram utilizados como correspondente à mesa de tensão (Kiehl, 1979).

* vedação do fundo dos anéis com tecido de nylon preso por um atilho de borracha e pesagem destes anéis;

* preenchimento dos anéis metálicos, de 68,7 mL de capacidade, com os substratos; a quantidade deve ser calculada através da densidade dos mesmos, para garantir a uniformidade de volume;

* colocação dos anéis em bandejas plásticas com água até $1 / 3$ de sua altura, para saturação, por 24 horas;

* retirada dos anéis da água;

* pesagem dos anéis. O volume de água contida na amostra neste momento corresponde ao ponto zero de tensão $(0 \mathrm{hPa})$, correspondendo à porosidade total;

* transferência dos anéis para o funil, previamente ajustado para tensão de 10 cm de coluna de água;

* permanência no funil até atingir equilíbrio (cerca de 48 horas); pesagem;

* retorno dos anéis ao funil ajustado para tensão de $50 \mathrm{~cm}$ de coluna de água;

* aguardar equilíbrio; pesagem;

* retorno dos anéis ao funil ajustado para tensão de $100 \mathrm{~cm}$ de coluna de água.

* aguardar equilíbrio; pesagem;

* secagem das amostras em estufa a $105^{\circ} \mathrm{C}$ até peso constante (ou 48 horas), para determinação dos teores de umidade e peso da matéria seca.

A construção das curvas de retenção de água foi efetuada com os valores de umidade volumétrica obtidos através dos percentuais de água retida para cada tensão.

De posse destes dados, foram obtidos os seguintes parâmetros:

${ }^{1}$ Comunicação pessoal. 2004. Informação de seu proprietário Alceu Silva 
Porosidade Total (PT): corresponde à umidade volumétrica presente nas amostras saturadas (tensão 0 )

$P T=\frac{[\text { Peso úmido (tensão } 0)-\text { Peso } \sec o(\text { estufa }]}{\text { Volume do anel }} * 100$

Espaço de Aeração (EA): representado pela diferença obtida entre a porosidade total e a umidade volumétrica na tensão de $10 \mathrm{~cm}$.

$E A=\frac{[\text { Peso úmido }(\text { tensão } 0)-\text { Peso úmido }(\text { tensão } 10]}{\text { Volume do anel }} * 100$

Água Facilmente Disponível (AFD): volume de água encontrado entre os pontos 10 e $50 \mathrm{~cm}$ de tensão.

$A F D=\frac{[\text { Peso úmido }(\text { tensão } 10)-\text { Peso úmido }(\text { tensão } 50]}{\text { Volume do anel }} * 100$

Água Tamponante (AT): a água volumétrica liberada entre 50 e $100 \mathrm{~cm}$ de tensão.

$A T=\frac{[\text { Peso úmido }(\text { tensão } 50)-\text { Peso úmido }(\text { tensão } 100]}{\text { Volume do anel }} * 100$

Água Disponível (AD): volume de água liberado entre 10 e $100 \mathrm{~cm}$ de tensão.

$A D=\frac{[\text { Peso úmido }(\text { tensão } 10)-\text { Peso úmido }(\text { tensão } 100]}{\text { Volume do anel }} * 100$

\section{Métodos para a caracterização química}

Valor de $p H$ - As leituras foram feitas em suspensões de substrato:água deionizada na proporção de 1:2,5 (v:v), através de potenciômetro. O método consiste em:

* colocar, em becker de $100 \mathrm{~mL}$, $20 \mathrm{~mL}$ da amostra (calculado segundo a densidade) e $50 \mathrm{~mL}$ de água deionizada;

* homogeneizar as suspensões com bastão de vidro logo após a colocação da água, e depois de 30 minutos e uma hora;

* fazer a leitura com potenciômetro previamente calibrado;

* lavar o eletrodo após cada leitura com água deionizada e secar em papel toalha.

Teor total de sais solúveis - O método utilizado foi o recomendado pela Federação dos Institutos para Pesquisas e Análises Agrícolas VDLUFA, na
Alemanha (Röber \& Schaller, 1985) e ALVA, na Aústria (Baumgarten, 2002). A condutividade do extrato, expressa como teor de $\mathrm{KCl}$, determina o Teor Total de Sais Solúveis (TTSS) de uma suspensão de substrato:água deionizada, na proporção 1:10 (peso:volume), através dos seguintes passos:

* colocar em frasco "snap-cap", $20 \mathrm{~g}$ de substrato e $200 \mathrm{~mL}$ de água deionizada;

* agitar por 3 horas em agitador mecânico;

* deixar em repouso até decantação das partículas;

* havendo necessidade, proceder a filtração das suspensões com papel de filtro ou centrifugação;

* fazer leitura em condutivímetro, da condutividade elétrica do material filtrado;

* realizar uma prova em branco para ajustes devido ao uso do papel filtro para a filtragem;

* expressar os resultados em gramas de $\mathrm{KCl}$ por litro de substrato, através dos seguintes cálculos:

$$
\begin{aligned}
& \operatorname{TTSS}\left(\mathrm{g} \mathrm{L}^{-1}\right)=\frac{\mathrm{X}^{*} \mathrm{C} * 56,312 *\left[\mathrm{D} \text {. úmida }\left(\mathrm{kg} \mathrm{m}^{-3}\right)\right] / 1000}{100.000} \\
& \text { sendo: }
\end{aligned}
$$

$\mathrm{X}=$ leitura do condutivímetro em Siemens $* 10^{-4}$

$\mathrm{C}=$ constante da célula do condutivímetro $=1$ para aparelhos com correção automática de temperatura

56,312 = fator de correção para expressar a condutividade em $\mathrm{mg}$ de $\mathrm{KCl} / 100 \mathrm{~g}$ de substrato, à temperatura de $25^{\circ} \mathrm{C}$.

$100.000=$ fator de conversão das unidades para $\mathrm{kg} \mathrm{m}^{-3}$ (= $\left.\mathrm{g} \mathrm{L}^{-1}\right)$

Todas as análises, físicas e químicas, foram realizadas em três repetições. As variáveis físicas foram analisadas pelo Programa Sigma Stat 3.2. As médias apresentadas para caracterização dos resíduos são as originais, não tendo sofrido nenhuma transformação.

\section{RESULTADOS E DISCUSSÃO}

Não houve diferença estatística en- tre as amostras para as características físicas estudadas. As amostras oriundas do litoral não diferiram daquelas obtidas no interior do Rio Grande do Sul, assim como, as amostras que sofreram hidrólise ácida não diferiram daquelas in natura, também as partes da planta (folhas, estipe e cartucho) não apresentaram diferenças significativas entre si (Figuras 1 e 2). O valor máximo assumido pelo coeficiente de variação foi de 21\% para a variável densidade seca, quando avaliada dentre as partes da planta, os demais valores de $\mathrm{CV}$ situaram-se abaixo de $10 \%$.

Os resultados das características físicas (DU, DS, PT e EA) encontram-se dentro das faixas consideradas adequadas para cultivo, indicando que os resíduos oriundos da extração do palmito da palmeira real australiana são adequados para uso como substrato para plantas. Entretanto, necessita-se verificar sua adequação ao cultivo em recipiente e a manutenção da estrutura física através da resistência à decomposição.

Os valores de densidade seca (entre 82 e $143 \mathrm{~g} \mathrm{~L}^{-1}$ ) são considerados baixos, sendo indicados para uso em bandejas, conforme Kämpf (2000) (Figura 1).

Todas as amostras apresentaram altos valores de porosidade total (entre 84 e $90 \% \mathrm{v})$, dentro da faixa de referência $(0,85 \% \mathrm{v})$ para substratos, segundo De Boodt et al. (1974) (Figura 2).

Todos os materiais têm altos valores de espaço de aeração (entre 0,48 e $0,64 \% \mathrm{v}$ ) quando comparados ao valor de referência internacional que é de $0,25 \% \mathrm{v}$, sugerido por De Boodt et al. (1974), embora Bunt (1973) e Verdonck \& Gabriels (1988) sugiram valores entre 0,10 e $0,15 \%$ v. Assim, inicialmente, este material pode ser indicado para produção de mudas pelo sistema de estaquia e/ou em sistemas com alta freqüência de regas. Considerando-se que a maioria dos materiais componentes de substratos têm baixos valores de espaço de aeração, torna-se promissor o uso destes resíduos como componentes em misturas e como condicionadores.

Já os resultados da água facilmente disponível e da água tamponante são insignificantes quando existentes, indicando que estes materiais não disponibilizam água para as plantas em cultivo 


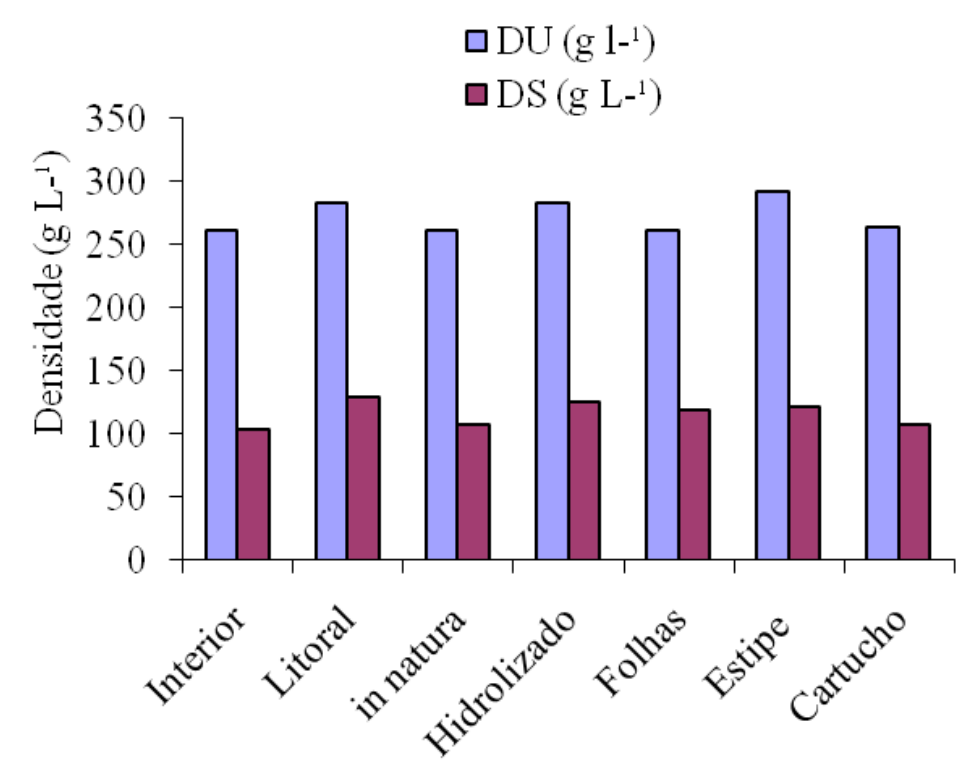

Figura 1. Densidade úmida e seca dos resíduos oriundos da indústria de conserva do palmito, proveniente da palmeira real australiana (Archontophoenix alexandrae), segundo o local de origem (interior e litoral), o tratamento recebido (in natura e hidrolizado) e parte da planta (folhas, estipe e cartucho) [humid and dry density of residues from the canning industry of palm heart (Archontophoenix alexandrae), according to the place of origin (inland and seaside), the received treatment (in natura and hydrolysed) and part of the plant (leaves, estip and cartridge - sheaths)]. Porto Alegre, FEPAGRO, 2005.

\section{(Figura 2).}

Os resultados das características químicas consideradas sugerem a realização de mais estudos para confirmá- los (Tabela 1). Todos os valores de $\mathrm{pH}$ estão fora das recomendações, ou muito altos como 8,83 do estipe in natura do interior, ou baixos (entre 1,83 das folhas hidrolisada do litoral e, 5,02 das folhas in natura do interior). No entanto, é possível verificar que a hidrólise ácida diminuiu todos os valores de $\mathrm{pH}$ dos materiais.

Gruszynski (2002) reuniu resultados de pesquisa do valor de $\mathrm{pH}$ recomendado por diversos autores para substratos, quando em cultivo de plantas ornamentais. Nela encontram-se referências de valor de $\mathrm{pH}$, em água, entre 5,4 e 6,8 para culturas diversas, à exceção de samambaias, bromélias, azaléias e coníferas que requerem $\mathrm{pH}$ entre 4,5 e 5,0.

A salinidade, em solos, é expressa como condutividade elétrica (CE) que expressa a capacidade do meio de conduzir eletricidade. Em substratos, a salinidade é expressa como teor total de sais solúveis (TTSS), que expressa a concentração de sais em determinado volume de substrato, calculada como $\mathrm{KCl}$.

Assim, em substratos, não basta observar o valor da $\mathrm{CE}$, mas considerar a densidade do material. Para o mesmo valor de $\mathrm{CE}$, maior será a salinidade no recipiente, quanto maior for a densidade do substrato.

Todos os valores de salinidade variam de médio $\left(1,06 \mathrm{~kg} \mathrm{~m}^{-3}\right.$ para estipe hidrolisado do interior) a extremamente alto $\left(6,60 \mathrm{~kg} \mathrm{~m}^{-3}\right.$ para estipe in natura

Tabela 1. Características químicas (valor de pH, condutividade elétrica (CE) e teor total de sais solúveis (TTSS) dos resíduos da indústria de conserva do palmito, segundo a origem (interior e litoral), tratamento (in natura e hidrolizada) e partes da planta (folhas, estipe e cartucho) [chemical characteristics ( $\mathrm{pH}$, electric conductivity $(\mathrm{CE})$ and total content of soluble salts (TTSS) of the waste of canning industry of heart of palm tree, according to the origin (inland and seaside), treatment (in natura and hydrolyzed) and parts of the plant (leaves, estipe and cartridge)]. Porto Alegre, FEPAGRO, 2005.

\begin{tabular}{|c|c|c|c|c|c|}
\hline Local & Tratamento & Partes da planta & $\mathrm{PH}\left(\mathrm{H}_{2} \mathrm{O}\right)$ & $\mathrm{CE}^{*}\left(\mathrm{dS} \mathrm{m} \mathrm{m}^{-1}\right)$ & TTSS $\left(\mathrm{kg} \mathrm{m}^{-3}\right)$ \\
\hline \multirow{6}{*}{ Interior } & \multirow{3}{*}{ In natura } & Folhas & 5,02 & 2,27 & 3,78 \\
\hline & & Estipe & 8,83 & 4,10 & 6,60 \\
\hline & & Cartucho & 4,89 & 2,07 & 3,01 \\
\hline & \multirow{3}{*}{ Hidrolizada } & Folhas & 2,44 & 0,90 & 1,45 \\
\hline & & Estipe & 2,51 & 0,62 & 1,06 \\
\hline & & Cartucho & 2,44 & 0,70 & 1,11 \\
\hline \multirow{6}{*}{ Litoral } & \multirow{3}{*}{ In natura } & Folhas & 4,20 & 1,28 & 1,86 \\
\hline & & Estipe & 4,26 & 1,00 & 1,38 \\
\hline & & Cartucho & 3,30 & 1,28 & 2,04 \\
\hline & \multirow{3}{*}{ Hidrolizada } & Folhas & 1,83 & 1,11 & 1,93 \\
\hline & & Estipe & 2,17 & 0,64 & 1,12 \\
\hline & & Cartucho & 2,06 & 0,88 & 1,55 \\
\hline
\end{tabular}

*diluição do extrato 1:10 substrato:água (volume:volume) [dilution of the extract 1:10 substrate:water (volume:volume)]. 


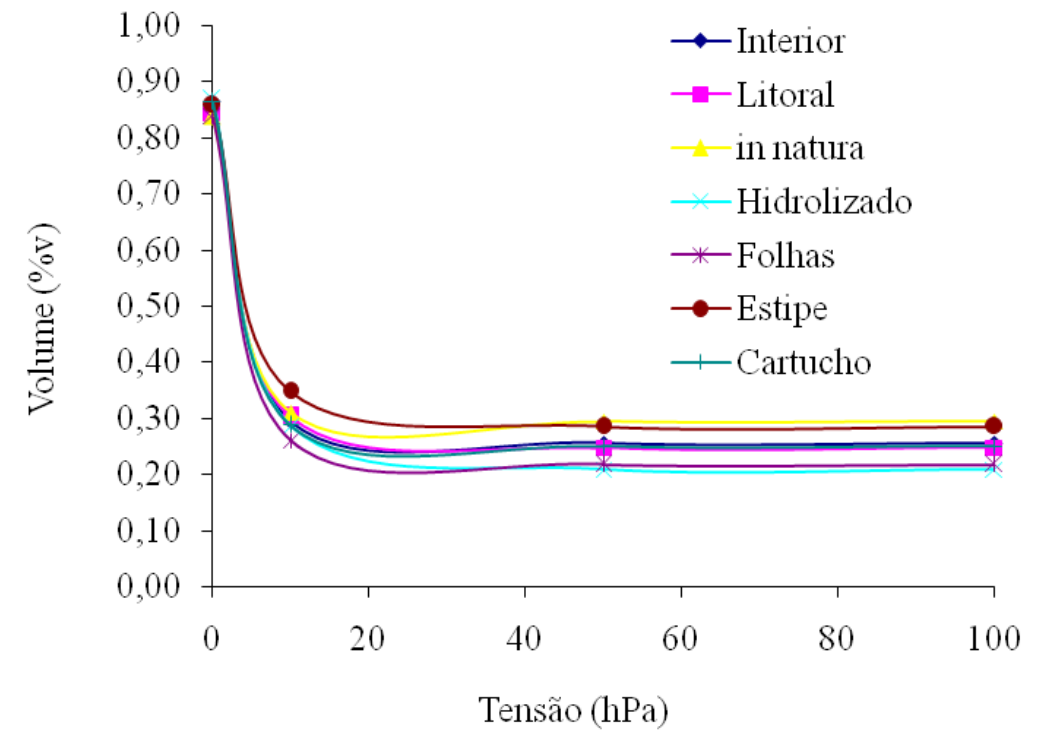

Figura 2. Curva de liberação de água dos resíduos oriundos da indústria de conserva do palmito, proveniente da palmeira real australiana (Archontophoenix alexandrae), segundo o local de origem (interior e litoral), o tratamento recebido (in natura e hidrolizado) e parte da planta (folhas, estipe e cartucho) [water release from the waste of canning industry of palm heart (Archontophoenix alexandrae), according to the place of origin (inland and seaside), the received treatment (in natura and hydrolysed) and part of the plant (leaves, estip and cartridge)]. Porto Alegre, FEPAGRO, 2005.

do interior) (Tabela 1). Estes resultados também indicam que a hidrólise diminui a condutividade elétrica do meio, e que as plantas do litoral têm menor salinidade do que as do interior. No entanto, como as amostras foram coletadas ao acaso, sem controle de possíveis tratamentos prévios por parte do produtor, estes resultados podem estar camuflados.

Segundo Kämpf (2000), a salinidade dos substratos pode ser classificada, em baixa $\left(<1,0 \mathrm{~kg} \mathrm{~m}^{-3}\right)$, média ou normal $\left(1,0-2,0 \mathrm{~kg} \mathrm{~m}^{-3}\right)$, alta $\left(2,0-4,0 \mathrm{~kg} \mathrm{~m}^{-3}\right)$, muito alta $\left(4,0-5,0 \mathrm{~kg} \mathrm{~m}^{-3}\right)$, extremamente alta $\left(5,0-7,0 \mathrm{~kg} \mathrm{~m}^{-3}\right)$ e tóxica $\left(>7,0 \mathrm{~kg} \mathrm{~m}^{-3}\right)$.

Os resíduos da indústria de conserva de palmito oriundos da palmeira real australiana, estudados neste trabalho, apresentam características físicas adequadas para uso como substrato para plantas, independente da sua origem, parte da planta, e se hidrolisadas ou in natura.

No entanto, os resultados das carac- terísticas químicas consideradas não são apropriados para a utilização dos resíduos da indústria de conserva de palmito como substrato para plantas e indicam a necessidade de novos estudos com resíduos provenientes de plantio controlado da palmeira real australiana.

\section{AGRADECIMENTOS}

À FINEP e à Fundação de Amparo à Pesquisa do Rio Grande do Sul (FAPERGS) pelo financiamento do projeto através do Programa de Apoio a Pesquisa nas Empresas - PAPPE (Edital FAPERGS 007-2004).

\section{REFERÊNCIAS}

BAUMGARTEN A. 2002. Methods of chemical and physical evaluation of substrates for plants. Documentos IAC 70: 07-15.

BUNT AC. 1973. Some physical and chemical characteristics of loamless pot-plant substrates and their relation to plant growth. Plant and Soil 38: 1954-1965.

De BOODT M; VERDONCK O. 1972. The physical properties of the substrates in horticulture. Acta Horticulturae 26: 37-44.

De BOODT M; VERDONCK O; CAPPAERT I. 1974. Method for measuring the waterrelease curve of organic substrates. Acta Horticulturae 37: 2054-2062.

FERMINO MH. 1996. Aproveitamento de residuos industriais e agrícolas como alternativas de substratos horticolas. Porto Alegre: UFRGSFA. 95p. (Tese mestrado).

GRUSZYNSKI C. 2002. Resíduo agro-industrial "casca de tungue" como componente de substrato para plantas. Porto Alegre: UFRGSFA. 100p (Tese mestrado).

GÜNTHER J. 1984. Analytics of substrates and problems by transmitting the results into horticultural practice. Acta Horticulturae 150: $33-40$.

HOFFMANN G. 1970. Verbindliche Methoden zur Untersuchung von TKS und Gärtnerischen Erden. Mitteilungen der VDLUFA 6: 129153.

KÄMPF AN. 2000. Produção Comercial de Plantas Ornamentais. Guaíba: Agropecuária. $254 \mathrm{p}$.

KIEHL EJ. 1979. Manual de Edafologia: Relações Solo-Planta. São Paulo: CERES. 263p.

LAMANNA D; CASTELNUOVO M; D'ANGELO G. 1991. Compost-based media as alternative to peat on ten pot ornamentals. Acta Horticulturae 294: 125-129.

MAAS EF; ADAMSON RM. 1975. Peat, bark and sawdust mixtures for nursery substrates. Acta Horticulturae 82: 147-151.

MINER AM. 1994. Substratos: propriedades y caracterizacion. Madrid: Ediciones MundiPrens. 172p.

MÜLLER. JJV. 2000. A demanda em substrato sob o ponto de vista dos usuários: na olericultura. In: KÄMPF, AN; FERMINO, MH (eds). Substrato para plantas: A base da produção vegetal em recipientes. Porto Alegre: Gênesis. $312 \mathrm{p}$.

RAJA HARUN RM; HALL DA; SZMIDT RAK; HITCHON GM. 1991. Melon cultivation in organic and inorganic substrates. Acta Horticulturae 294: 105-108.

RÖBER R; SCHALLER K. 1985. Pflanzenernährung im Gartenbau. Stuttgart: Ulmer. 352p.

SCHMILEWSKI GK. 1991. Quality control and use of composted organic wastes as components of growing media in the Federal Republic of Germany. Acta Horticulturae 294: 89-98.

VERDONCK O. 1984. Reviewing and evaluation of new materials used as substrates. Acta Horticulturae 150: 467-473.

VERDONCK O; GABRIELS R. 1988. Substrate requeriments for plantas. Acta Horticulturae 221: 19-23.

WALLER PL; WILSON GCS. 1984. Evaluation of growing media for consumer use. Acta Horticulturae 150: 51-58.

WORRALL RJ. 1978. The use of composted wood waste as a peat substitute. Acta Horticulturae 82: 79-86. 